\title{
Advanced TMJ Imaging-A Review
}

\author{
Malusare PC ${ }^{1}$, Das D ${ }^{1}$, Navalkar A ${ }^{1}$, Sali S ${ }^{2}$ and Sridharan $\mathrm{G}^{3}$ \\ ${ }^{1}$ Department of Oral medicine \& Radiology GD Pol Foundation YMT Dental College, \\ India \\ ${ }^{2} \mathrm{BDS}$, Private practice, India \\ ${ }^{3}$ Department of Oral Pathology, GD Pol Foundation's YMT Dental College, India
}

\section{Review Article \\ Volume 3 Issue 4}

Received Date: August 07, 2019

Published Date: August 29, 2019

DOI: $10.23880 /$ crij-16000153

*Corresponding author: Pratik Malusare, Senior Lecturer, Oral medicine \& Radiology Department ,GD Pol Foundation YMT Dental College, Kharghar, Navi Mumbai-410210, India, Tel: 91-8369030834; Email: pratsmalu@gmail.com

\begin{abstract}
Temporomandibular joint (TMJ) diseases and disorders refer to a complex and poorly understood set of conditions, manifested by pain in the area of the jaw and associated muscles, and limitations in the ability to make the normal movements of speech, facial expression, eating, chewing, and swallowing. Knowledge of the different imaging tests and their appropriate indications is crucial to establish the diagnosis of temporomandibular disorders. Definitive and rational diagnoses or treatments can only be achieved through a comprehensive understanding of the causative factor, predisposing factors, and pathogenesis of TMJ diseases. Radiographic examination forms an integral component of the clinical assessment routine in patients suffering from temporomandibular disorders (TMD). Imaging of the TMJ is continuously evolving with advancement of imaging technologies. Latest imaging modalities is making easier for those clinicians in evaluation of hard tissues of the temporomandibular joint complex in patients with orofacial pain. They have greatly contributed to the understanding of diseases of the temporomandibular joint. The present paper attempts to highlight the various advance imaging modalities for TMJ diagnosis.
\end{abstract}

Keywords: Temporomandibular Joint; Magnetic Resonance Imaging; Imaging; Computed Tomography

Abbreviations: TMJ: Temporomandibular Joint; TMD: Temporomandibular Disorders; AAOMR: American Academy of Oral and Maxillofacial Radiology; MRI: Magnetic Resonance Imaging; CBCT: Cone Beam computed tomography; SPECT: Single-Photon Emission Computed Tomography; PET: Positron Emission Tomography; MDP: Methylene Diphosphonate.

\section{Introduction}

The temporomandibular joint (TMJ) is a synovial joint whose common features are represented by a disc, two bones, a fibrous capsule, intra-articular fluid, a synovial membrane and ligaments [1]. However, the Temporomandibular Disorder (TMD) is commonly 


\section{Clinical Radiology \& Imaging Journal}

observed with structural changes and functional derangements of the TMJ stomatognathic own and adjacent structures such as chewing muscles, ligaments, teeth and periodontal tissue [2,3]. Radiological investigations are of paramount importance in the diagnostic assessment of a patient with TMD. The American Academy of Oral and Maxillofacial Radiology (AAOMR) has established the rationale for image selection for diagnosis, treatment planning and follow up of a patient with conditions affecting the TMJ (parameter 2) [4].

Although the clinical examination is the most important step in the diagnosis of TMJ pathology, special imaging techniques are needed due to the complex anatomy and pathology. One important thing to consider when imaging the TMJ is the interpretation of the joint function, which can be accomplished by comparing the condyle in the closed and opened mouth position. Conventional radiographic TMJ projections like transpharyngeal, transcranial, panoramic radiograph, conventional tomographic sections of TMJ may be adequate in a number of clinical situations. But there are bony alterations that occur in these disorders like erosions, osteophytes, pneumatization of articular eminence that are difficult to be detected in conventional radiographs due to overlapping of the anatomic structures [5]. This warrants the use of advanced imaging modalities like, computed tomography, Magnetic Resonance Imaging (MRI), Cone Beam computed tomography (CBCT), Ultrasonography and Nuclide imaging.

\section{Computed Tomography}

The use of CT in diagnosing TMJ disorders dates back to the late 1980s [6]. CT is considered to be the best method for assessing osseous pathologic conditions of TMJ. It allows a multi planar reconstruction (sagittal, axial, and coronal) of TMJ structures, obtaining 3D images in closed and opened-mouth positions. Basically, any CT examination of the TMJ should focus on the following: intactness of the cortex, normal size and shape of the condyles and their centered position in the fossa, the adequate joint spaces, centric relation loading zone. CT is the optimal imaging modality to identify and characterize the calcified intra-articular fragments [7]. Westesson PL, et al. found a sensitivity of $75 \%$ and specificity of $100 \%$ for the diagnosis of condylar osseous changes [8]. Changes in the shape and location of the loading zone can also be seen on CT. CT is the main radiological investigation for tumors, growth development anomalies and fractures. Regarding the visualization of the soft tissues of TMJ (disc, synovial membrane, ligaments, lateral pterygoid muscle), CT is not used as a primary diagnostic method. Arthrography is combined with computer tomography, which enhances the accuracy of the diagnosis of internal TMJ disorders. On CT scans, the position and the shape of the mandibular condyle in the glenoid fossa is well seen, though some authors suggest that this reference is not a precise sign of disc pathology [9-11]. CT scanning is performed when fine detail in bone anatomy is of primary importance. Three-dimensional CT plays an important role in the assessment of osseous deformities of the jaw.

\section{MRI}

MRI is an imaging technique in which not only bone, but also soft tissue structures can be reproduced in detail through the use of static and dynamic magnetic fields. In the 1980s MRI was used for the first time introducing the so-called surface spool for examination and featuring of TMJ structures [12]. It is a unique imaging modality that produces cross sectional multiplanar images without using ionizing radiation. Imaging of soft tissues is superior to that of computed tomography, less invasive that arthrography, and more reliable than radiography Despite the superior resolution of CT and limited visualization of cortical bone by MRI, most osseous pathology is accurately depicted. Intra articular abnormalities are readily visible on MRI images, providing further information not available with other imaging modalities [13-14]. For optimal imaging of the TMJ, small bilateral surface coils with small field of view are used to achieve higher signal to noise ratio and simultaneous bilateral acquisition. Closed mouth coronal and axial T1 sequences are needed to evaluate the overall anatomy and bone marrow as well as the adjacent soft tissues to exclude other adjacent pathology. An MR image is produced from signals coming from the hydrogen nuclei, or protons, in the body. The contrast of the image is provided by differences in signal intensity from protons in different tissues.

MRI is preferably appropriate to assess variations of the disk. Oblique sagittal and coronal images can be oriented to the condyle, but are unnecessary to demonstrate internal derangements. T1-weighted sagittal images are the cornerstone of the TMJ examination; the anatomy is clearly depicted, and the imaging plane is optimal for assessing articular disk position. T2-weighted images are useful for detecting degenerative periarticular changes and the presence of a joint effusion [15]. MRI evaluation of the TMJ begins with the determination of disc position in the sagittal plane with the mouth closed. 


\section{Clinical Radiology \& Imaging Journal}

Normally, the posterior band lies at the 10:00 to $12: 00$ position in relation to the condylar head. If the posterior band is positioned more anteriorly, it is said to be anteriorly displaced. If the posterior band is noted to extend beyond either the medial or lateral pole of the condylar head, the displacement is said to have a rotational component, which is described as anteromedial or anterolateral displacement. The next step is to determine the position of the disc on the coronal images. Rarely, coronal images may reveal pure sideways displacement of the disc, a finding that may or may not be suspected, based on the sagittal images. The next step is to determine the disc position with the mouth open.

Pain or suboptimal effort may reduce the maximum degree of opening. Normally, in the maximal open mouth position, the condylar head should lie directly inferior or slightly anterior to the articular eminence, and if the disc is seen in its expected position relative to the condylar head and articular eminence, it is said to be reduced, or recaptured. When a disc remains anteriorly positioned, it is called anterior displacement without reduction or recapture. The advantages of MRI are: a) it is noninvasive, b) it requires no ionizing radiation for image acquisition, c) it permits a direct visualization of the disk and joint structures, and d) multiplanar imaging is readily obtained and more easily interpretable [16]. The disadvantages of MRI are: a) It is more expensive examination and is contradicted in patients who are pregnant, or who have pacemakers, intracranial vascular clips, or metal particles in vital structures. b) Some patients may not be able to tolerate the procedure because of claustrophobia or an inability to remain motionless [17].

\section{Computed Beam Computed Tomography}

CBCT is a recent technology, which was first applied for angiography in the early 1980s and then later gradually used for other applications [18]. New generation CBCT utilize less expensive equipment, and expose the patient to approximately $20 \%$ of the radiation of helical CT [19]. Conventional CT equipment using a fan shaped $X$ ray beam captures a series of axial plane slices or from a continuous spiral motion over the axial plane. A CBCT machine, on the other hand, uses a cone-shaped beam and a reciprocating solidstate flat panel detector, which rotates once around the patient (180-360 degrees, covering the defined anatomical volume (complete dental/ maxillofacial volume or limited regional area of interest) rather than slice-by-slice imaging found in conventional CT. Designation of this equipment is not based on the concept of sectional images but in computer processing of a single rotational scanning of the region of interest.

CBCT has specialized imaging capabilities for assessing osteoarthritic changes in detail, and for accurately calculating joint space [20]. The RDC/TMD validation project recommends the use of $\mathrm{CT}$ both in clinical and research setting since it is superior to the aforementioned modalities in showing osseous abnormalities of the TMJ [11] High cost of the equipment, infrastructural demands and concerns over radiation dose to the patients have confined the use of medical CT largely to the hospital settings [21]. According to Tsiklakis K, et al. CBCT should be used instead of CT because the dose of radiation to which the patient is exposed is much lower. Furthermore, CBCT is superior to CT for visualizing bone changes in the TMJ, analysing lateral slices in isolation and combining coronal and lateral slices [22]. A large body of literature has been published in recent times due to the fact that CBCT has inspired research in TMJ imaging [23]. The reconstructed images are of high diagnostic quality, the examination time is shorter, and patient dose is lower than that with conventional CT. It may therefore be considered as the imaging technique of choice when investigation of bony changes of the TMJ is the task at hand.

\section{Ultrasonography}

Ultrasound imaging of the joint and surrounding tissues seems to be a reasonable direction in which TMD diagnostics should proceed. It is a cheap, non-invasive, and quick examination technique, widely available in most healthcare institutions. The first reports of TMJ sonography date back to 2000 . It uses currently available types of ultrasonic equipment with a linear scanning transducer of 7.5-12 $\mathrm{MHz}$ frequency, which makes it possible to depict the narrow space of the jaw joint and the position of the joint disc and it reveals fluid or ligament adhesion [24]. The principle of ultrasonography is based on the fact that ultrasonic sound waves emitted by a device (transducer), travel through the tissue against which they are aimed, and are partly reflected on transiting through dissimilar anatomical structures. The reflected sound waves are then read by the same emitting device, and translated into images [25].

During an ultrasound of the temporomandibular joint, a linear probe is used, which is positioned transversely or longitudinally relative to the zygomatic arch, and then tilted to obtain the best view. During the examination, the patient remains in a prone position with their mouth either closed or maximally open [26]. The transducer is 


\section{Clinical Radiology \& Imaging Journal}

placed over the joint parallel to the long axis of the mandible. The joint disk is scanned on the screen as a thin homogeny hypo, as far as the isoechogen strip adjacent to the condylar border. The condylar borders and articular eminence are seen as hyperechogen lines. A study published in 2006 by Jank S, et al., which was conducted on 100 patients and which compared the techniques of ultrasound and MRI in terms of detecting degenerative changes, joint effusion, and disc displacement obtained very good results [27]. Dupuy-Bonafé I, et al. do not recommend the use of ultrasound in the diagnosis of TMD as in their research they obtained low sensitivity but high specificity in the diagnosis of disc displacement [28]. Kaya $\mathrm{K}$, et al., on the other hand, obtained high sensitivity and accuracy, but low specificity. They concluded that ultrasound is useful for the diagnosis of disc displacement but unfortunately not very effective in excluding disorders in a healthy temporomandibular joint.

Although ultrasound can detect disc displacement, it cannot determine the type of displacement [29]. Ultrasonographic examination is a procedure which greatly depends on the operator's skill and experiences. Differences of opinion among researchers may result from the lack of standardization in the procedures related to conducting the examination: starting from the selection of the transducer, its settings, and the mouth opening position; and ending with interpreting the results using either the direct or indirect method. A probe with a higher frequency enables a better visualization of the tissues, with high frequency ultrasound using a probe with a frequency equal to or higher than $12 \mathrm{MHz}$ [30]. USG is a promising modality but its sensitivity, accuracy and positive predictive value will have to be improved in the future, through the use of higher-resolution equipment [31].

\section{Nuclide Imaging}

Nuclear medicine deals with diagnostics and therapy using radioactive isotopes emitting beta or gamma radiation. Radiopharmaceuticals are pharmaceuticals containing radioactive isotope as tracer and a ligand, i.e., a molecule, chemical compound or cell (e.g. granulocyte) that has an affinity towards a tissue or organ [32]. Since the introduction of nuclear imaging, many technological advances have been made to expand the clinical and research applications of nuclear imaging; one of them is the improvement of image resolution of the scans. Among other technical advances are those: (1) a variety of radiopharmaceuticals has been synthesized and made widely available for clinical use; (2) sophisticated computer-assisted imaging equipment has been developed; and (3) effective imaging protocols have been identified to meet a variety of research, diagnostic, and treatment planning objectives [33]. Registration of radiation can be performed by means of a single static gamma camera (also known as a scintillation camera), one or more rotating gamma cameras or multi headed gamma cameras. Depending on the type of registration device, the imaging methods are divided into scintigraphy, single-photon emission computed tomography (SPECT) and positron emission tomography (PET).

\section{Scintigraphy}

Scintigraphy aids in discovering the early changes in the TMJ apparatus which may also result in joint disc abnormalities. Bone scintigraphy is performed using osteotropic tracers, most commonly methylene diphosphonate (MDP) linked with radioactive technetium $(99 \mathrm{mTc})$, which is injected into a vein (arm, hand or foot). MDP targets bone tissue, and its uptake depends on bone mineralisation, collagen content, vascularisation and bone remodelling. Forming of hydroxyapatite crystals in the areas of production of new osteoid tissue by osteoblasts leads to an increased uptake of tagged MDP; thus these areas accumulate more radioactive tracer and show up as "hot" [34]. Planar scintigraphy of TMJ is performed typically in anterior, posterior, right lateral and left lateral projections with a 500,000 count per each image [35-36]. The use of bone scintigraphy in TMJ diagnostics is not common in comparison with other diagnostic imaging techniques. [37] Kim JH, et al. [38] evaluated usefulness of bone scintigraphy in diagnostics of TMJ osteoarthritis. There were significant differences in uptake ratios between the osteoarthritis and non-osteoarthritis groups thus leading to a conclusion that bone scans may help to diagnose osteoarthritis when uptake ratios are increased. Open-mouth bone scintigraphy was proved to be better than closed-mouth bone scan in patients with temporomandibular osteoarthritis as it is difficult to differentiate physiologic bone uptake in a condyle and glenoid fossa of temporal bone in closed-mouth position [39].

\section{Single-Photon Emission Computed Tomography}

SPECT is based on registration of emission of radiation by means of a rotating gamma camera to obtain projections from multiple angles. It uses the same radiopharmaceuticals as scintigraphy, but the acquired images are more precise regarding localisation of areas of tracer uptake. The temporomandibular joint is ideal for 


\section{Clinical Radiology \& Imaging Journal}

what is called SPECT, because it is a quite small joint situated close to the skull base and paranasal sinuses. So SPECT can, unlike the double-dimension featuring, present TMJ separately from the parts of high bone density. The radionuclide examination sensitivity is high, its specificity is however low. Any inflammation, trauma or tumors increase the local isotope concentration. For this reason many studies state that radionuclide examination is relevant only as a screening method [6]. The following applications of SPECT in TMJ imaging were reported [32]:

a) Unilateral condylar hyperplasia

b) Bone tracer uptake in patients suffering from TMJ pain

c) Osteoarthritis

d) Quantitative evaluation of temporomandibular joint disorder (TMD)

e) Evaluation of the effects of functional orthopaedic treatment of TMJ

\section{Positron Emission Tomography}

Positron Emission Tomography is a nuclear medicine imaging technique, which provides cross-sectional data based on the 3-dimensional localization of positrons emitted by radiotracers. When compared with scintigraphy, in addition to its cross-sectional nature, PET has the advantages of higher detection efficiency and spatial resolution [40]. The use of PET scans as an imaging modality has been increasing and is helpful in evaluating metabolic reactions in the body [41]. PET data reflects the distribution of the radiotracer in the area being imaged, but does not provide structural information relating to the anatomy of the patient. Rather, PET provides functional information based on the interaction of the radiotracers at the molecular level in relation to physiological events. In order to facilitate anatomic localization of areas of increased radiotracer uptake, PET is usually combined with computed tomography, which provides structural information of the patient [42]. Indications for PET scanning of TMJ area are mostly related to diagnostics and treatment follow-up of malignant tumours. They include early diagnostics of neoplasms; staging of some tumours, e.g. lymphoma; planning of radiotherapy; and location of an unknown primary tumour with known metastases, response to treatment, follow-up and differentiation between recurrent tumour and post-treatment lesions induced by, e.g. radiotherapy [32].

The first study to evaluate the clinical utility of FDGPET/CT in patients with TMD was published in 2013 by Lee JW, et al. [35]. They concluded that PET/CT showed high TMJ uptake rations in patients with osteoarthritis, while accuracy and sensitivity were higher than in conventional bone scintigraphy. Suh MS, et al. [36] investigated patients with temporomandibular joint disorder (TMD) by means of PET-CT with 8 F-sodium fluoride $(\mathrm{NaF})$ as tracer and found out that this imaging modality was useful in arthralgia TMJ and TMD osteoarthritis and a correlation with the patients' response to splint therapy was ascertained.

\section{Conclusion}

Reliable case history and the patient's clinical examination are usually insufficient to base exact diagnosis of temporomandibular defects on. Several radiographic methods are used to assess the TMJ, an area that is difficult to be imaged due to factors like superimposition of adjacent structures and morphological variations. The complexity of the TMD however, demands a clear and precise image of the region for effective management of the patients. Although CT has been traditionally viewed as a modality for assessing only bony pathology and calcification of the TMJs, with attention to technique and interactive reading with a DICOM viewer, it is an excellent modality for viewing the full range of TMJ pathology and can be used instead of MRI when MRI is contraindicated or not accessible. The MRI helps in the diagnostic approach of processes related to TMJ, in which the disc-related disorders and joint mobility are the most important, followed by degenerative changes. It is the only exam that allows the identification of the disc, soft tissue, cortical bone contour and bone marrow of the mandibular condyle, featuring the complete form of joint disorders and displays with best advantage the soft tissue components of the TMJ as well as bone marrow abnormalities, and is considered the golden standard in the evaluation of this joint.

CBCT provides a definite advantage over other techniques due to its low radiation dose to patient, smaller equipment and ability to provide multiplanar reformation and 3D images. There is promising research in the field of CBCT in TMJ imaging. Ultrasonography is a noninvasive and inexpensive diagnostic procedure that has been shown to be accurate for the diagnosis of articular disorder and joint effusion. For this reason it can be suggested for the evaluation of TMJ disorders. Nuclear medicine offers researchers a non-invasive and sensitive method for studies involving inflammations of oral structures, tumors, trauma, bone healing, and the temporomandibular joint. Its great advantage mainly consists in the possibility of depicting dynamic joint structures, particularly the condyle line and the joint disc position. Understanding of the TMJ anatomy, 


\section{Clinical Radiology \& Imaging Journal}

biomechanics, and the imaging manifestations of diseases is important to accurately recognize and manage these various pathologies.

\section{References}

1. Okeson JP (2008) Management of Temporomandibula $r$ disorders and occlusion. $6^{\text {th }}$ (Edn.), St. Louis: Mosby, pp: 5-21.

2. Guimarães JP, Ferreira LA (2012) Atlas de diagnóstico por imaginologia das desordens temporomandibulare s, Editora UFJF, Juiz de Fora Brazil.

3. Gatchel RJ, Stowell AW, Wildenstein L, Riggs R, Ellis E 3rd (2006) Efficacy of an early intervention for patients with acute temporomandibular disorderrelated pain: a one-year outcome study. J Am Dent Assoc 137(3): 339-347.

4. Scarfe WC, Farman AG, Sukovic P (2006) Clinical applications of cone-beam computed tomography in dental practice. J Can Dent Assoc 72(1): 75-80.

5. Marques AP, Perrella A, Arita ES, Pereira MF, Cavalcanti MG (2010) Assessment of simulated mandibular condyle bone lesions by cone beam computed tomography. Braz Oral Res 24(4): 467-474.

6. Tvrdy P (2007) Methods of imaging in the diagnosis of TMJ Disorders. Biomed Pap Med Fac Unic Palacky Olomouc Czech Repub 151(1): 133-136.

7. Malusare PC, Navalkar A, Das D, Soman BP (2018) Clinical and radiological findings of temporomandibular joint synovial chondromatosis perforating the glenoid fossa: A rare case report. J Oral Maxillofac Radiol 6(3): 55-58.

8. Westesson PL, Katzberg RW, Tallents RH, SanchezWoodworth RE, Svensson SA (1987) CT and MR of the temporomandibular joint: comparison with autopsy specimens. AJR 148(6): 1165-1171.

9. Pullinger AG, Hollender L, Solberg WK, Petersson A (1985) A tomographic study of mandibular condyle position in an asymptomatic population. J Prosthet Dent 53(5): 706-713.

10. Blaschke DD, Blaschke TJ (1981) Normal TMJ bony relationships in centric occlusion. J Dent Res 60(2): 98-104.

11. Paknahad M, Shahidi S, Iranpour S, Mirhadi S, Paknahad M (2015) Cone-Beam Computed
Tomographic Assessment of Mandibular Condylar Position in Patients with Temporomandibular Joint Dysfunction and in Healthy Subjects. Int J Dent 2015: 301796.

12. Tvrdy P (2007) Methods of Imaging in the Diagnosis of Temporomandibular Joint Disorders. Biomed Pap Med Fac Univ Palacky Olomouc Czech Repub 151(1): 133-136.

13. Edelman RR, Hesselink J, Zlatkin M (1990) Clinical Magnetic Resonance Imaging: 3-Volume Set $3^{\text {rd }}$ (Edn.), Philadelphia, WB Saunders.

14. Roth C, Ward RJ, Tsai S, Zolotor W, Tello R (2005) MR Imaging of the TMJ: A Pictorial Essay. Applied Radiology 34: 9-16.

15. Hayt MW, Abrahams JJ, Blair J (2000) Magnetic resonance imaging of the temporomandibular joint. Top Magn Reson Imaging 11(2): 138-146.

16. Krishnamoorthy B, Mamatha N, Kumar VA (2013) TMJ imaging by CBCT: Current scenario. Ann Maxillofac Surg 3(1): 80-83.

17. White SC, Pharoah MJ. Oral Radiology Principles and interpretation $5^{\text {th }}$ (Edn.), Petrikowski CG.

18. Pauwels R, Araki K, Siewerdsen JH, Thongvigitmanee SS (2015) Technical aspects of dental CBCT: state of the art. Dentomaxillofacial Radiol 44(1): 20140224.

19. Quereshy FA, Savell TA, Palomo JM (2008) Applications of cone beam computed tomography in the practice of oral and maxillofacial surgery. J Oral Maxillofac Surg 66(4): 791-796.

20. Alexiou K, Stamatakis H, Tsiklakis K (2009) Evaluation of the severity of temporomandibular joint osteoarthritic changes related to age using cone beam computed tomography. Dentomaxillofac Radiol 38(3): 141-147.

21. Ahmad M, Hollender L, Anderson Q Kartha K, Ohrbach R, et al. (2009) Research diagnostic criteria for temporomandibular disorders (RDC/TMD): development of image analysis criteria and examiner reliability for image analysis. Oral Surg Oral Med Oral Pathol Oral Radiol Endod 107(6): 844-860.

22. dos Anjos Pontual ML, Freire JS, Barbosa JM, Frazão MA, dos Anjos Pontual A (2012) Evaluation of bone changes in the temporomandibular joint using cone beam CT. Dentomaxillofac Radiol 41(1): 24-29. 


\section{Clinical Radiology \& Imaging Journal}

23. Tecco S, Saccucci M, Nucera R, Polimeni A, Pagnoni M, et al. (2010) Condylar volume and surface in Caucasian young adult subjects. BMC Med Imaging 10: 28.

24. Westesson PL (1993) Reliability and validity of imaging diagnosis of temporomandibular joint disorder. Adv Dent Res 7(2): 137-151.

25. Melis M, Secci S, Ceneviz C (2007) Use of ultrasonography for the diagnosis of temporomandibular joint disorders: a review. Am J Dent 20(2): 73-78.

26. Klatkiewicz T, Gawriołek K, Pobudek Radzikowska M, Czajka-Jakubowska A (2018) Ultrasonography in the Diagnosis of Temporomandibular Disorders: A MetaAnalysis. Med Sci Monit 24: 812-817.

27. Jank S, Emshoff R, Norer B, Missmann M, Nicasi A, et al. (2005) Diagnostic quality of dynamic highresolution ultrasonography of the TMJ--a pilot study. Int J Oral Maxillofac Surg 34(2): 132-137.

28. Dupuy-Bonafé I, Picot MC, Maldonado IL, Lachiche V, Granier I, et al. (2012) Internal derangement of the temporomandibular joint: is here still a place for ultrasound? Oral Surg Oral Med Oral Pathol Oral Radiol 113(6): 832-840.

29. Kaya K, Dulgeroglu D, Unsal-Delialioglu S, Babadag M, Tacal T, et al. (2010) Diagnostic value of ultrasonography in the evaluation of the temporomandibular joint anterior disc displacement. J Craniomaxillofac Surg 38(5): 391-395.

30. Habashi H, Eran A, Blumenfeld I, Gaitini D (2015) Dynamic high-resolution sonography compared to magnetic resonance imaging for diagnosis of temporomandibular joint disk displacement. J Ultrasound Med 34(1): 75-82.

31. Landes CA, Goral WA, Sader R, Mack MG (2007) Three dimensional versus two-dimensional sonography of the temporomandibular joint in comparison to MRI. Eur J Radiol 61(2): 235-244.

32. Różyło-Kalinowska I (2019) Nuclear Medicine in TMJ Imaging. In: Rozylo-Kalinowska I, Orhan K (Eds.), Imaging of the Temporomandibular Joint, Springer, Cham: 247-254.

33. Matteson SR, Deahl ST, Alder ME, Nummikoski PV (1996) Advanced Imaging Methods. Crit Rev Oral Biol Med 7(4): 346-395.
34. Choi BH, Yoon SH, Song SI, Yoon JK, Lee SJ, et al. (2016) Comparison of diagnostic performance between visual and quantitative assessment of bone scintigraphy results in patients with painful temporomandibular disorder. Medicine (Baltimore) 95(2): 2485.

35. Lee JW, Lee SM, Kim SJ, Choi JW, Baek KW (2013) Clinical utility of fluoride-18 positron emission tomography/CT in temporomandibular disorder with osteoarthritis: comparisons with 99m Tc-MDP bone scan. Dentomaxillofac Radiol 42(2): 29292350.

36. Suh MS, Lee WW, Kim YK, Yun PY, Kim SE (2016) Maximum standardized uptake value of $(99 \mathrm{~m})$ Tc Hydroxymethylene diphosphonate SPECT/CT for the evaluation of temporomandibular joint disorder. Radiology 280(3): 890-896.

37. Epstein JB, Rea A, Chahal O (2002) The use of bone scintigraphy in temporomandibular joint disorders. Oral Dis 8(1): 47-53.

38. Kim JH, Kim YK, Kim SG, Yun PY, Kim JD, et al. (2012) Effectiveness of bone scans in the diagnosis of osteoarthritis of the temporomandibular joint. Dentomaxillofac Radiol 41(3): 224-229.

39. Park KS, Song HC, Cho SG, Kang SR, Kim J, et al. Openmouth bone scintigraphy is better than closed-mouth bone scintigraphy in the diagnosis of temporomandibular osteoarthritis. Nucl Med Mol Imaging 50(3): 213-218.

40. Bushberg JT, Seibert JA, Leidholdt EM, Boone JM (2011) The Essential Physics of Medical Imaging. $3^{\text {rd }}$ (Edn.), Lippincott Williams and Wilkins, Philadelphia, PA.

41. Keyes JW Jr, Harkness BA, Greven KM, Williams DW 3rd, Watson NE Jr, et al. (1994) Salivary gland tumors: pretherapy evaluation with PET. Radiology 192(1): 99-102.

42. Spriet M, Willcox JL, Culp WTN (2019) Role of Positron Emission Tomography in Imaging of Nonneurologic Disorders of the Head, Neck, and Teeth in Veterinary Medicine. Front Vet Sci 6: 180. 